\title{
CRITERIOS $Y$ CONSIDERACIONES METODOLÓGICAS $Y$ TECNOLÓGICAS A TENER EN CUENTA EN EL DISEÑO E IMPLEMENTACIÓN DEL PROTOCOLO IEC 61850 EN LA AUTOMATIZACIÓN Y PROTECCIÓN DE SISTEMAS DE POTENCIA ELÉCTRICA
}

\section{METHODOLOGICAL CONSIDERATIONS AND CRITERIA (TECHNOLOGIES TO BE CONSIDERED) WHEN DESIGNING AND IMPLEMENTING THE IEC 61850 PROTOCOL FOR AUTOMATION AND PROTECTION OF ELECTRIC POWER SYSTEMS}

\begin{abstract}
The present paper discusses and analyzes the elements involved in Ethernet networks when such networks are implemented over electric-power systems that comply with standard IEC 61850. The concept behind intelligent networks (IN) is currently drawing a lot of attention within the electric power industry around the world and, in such a context, a proper understanding of this standard constitutes the first step towards the so called intelligent power distribution network. Hence, one of the main goals of this paper is to offer an insight into the structure and potential benefits of the IEC 61850 standard. Additionally, the paper presents some important considerations and criteria that must be included in the design of an Ethernet network to operate over an electric-power system.
\end{abstract}

Key words: GOOSE, IEC 61850, RSTP, HSR PRP, latency, IED, MMS.

\section{RESUMEN}

En este artículo se estudia y analiza los elementos que se involucran en redes Ethernet implementadas en sistema eléctricos desarrollados con la norma IEC 61850. El concepto de redes inteligentes (RI) está actualmente capturando la atención del sector eléctrico en todo el mundo y la norma es el primer avance para poder llegar a lo que se denomina red de distribución de energía eléctrica inteligente, por lo que uno de los objetivos principales de este escrito es dar a conocer la estructura y beneficios de la norma IEC 61850. Adicionalmente se presentan criterios y consideraciones que se deben tener en cuenta en el diseño de una red Ethernet para sistemas de potencia eléctrica.

Palabras claves: GOOSE, IEC 61850, RSTP, HSR PRP, latencia, IED, MMS.
Juan Carlos Carreño Pérez Especialista en Teleinformática Universidad Distrital Francisco José de Caldas

juan_carlos_carreno@hotmail.com Bogotá, Colombia

Danilo Alfonso López Sarmiento Magister en Ciencias de la Información y las Comunicaciones

Docente planta de la Universidad Distrital Francisco José de Caldas dalopezs@udistrital.edu.co Bogotá, Colombia

\section{Octavio José Salcedo Parra}

Magister en Ciencias de la Información y las Comunicaciones

Docente planta de la Universidad Distrital Francisco José de Caldas ojsalcedop@udistrital.edu.co Bogotá, Colombia

Tipo: Artículo de investigación

Fecha de Recepción: Enero 12 de 2012 Fecha de Aceptación: Marzo 9 de 2012 


\section{NOMENCLATURA Y ACRÓNIMOS}

GOOSE (generic object oriented substation event), IEC 61850 (estándar de redes y sistemas de comunicación en subestaciones"), LAN (red de área local), IGMP (protocolo de mensajes de grupos de Internet), MAC (control de acceso al medio), MMS (especificación de mensajes de fabricación), OSI (interconexión de sistemas abiertos), RSTP (protocolo de árbol de expansión rápido), SNMP (protocolo simple de administración de redes), SNTP (simple Network Time Protocol), TCP (protocolo de control de transporte), HSR (high availability seamless redundancy), PRP (protocolo de redundancia paralela), UDP ( protocolo de datagrama de usuario), IED (dispositivos electrónicos inteligentes).

\section{INTRODUCCIÓN}

La norma IEC 61850 ofrece soluciones a los requerimientos de los modernos sistemas de automatización y protección de sistemas de potencia eléctrica, en la medida que permite integrar en una sola red y protocolo, los distintos niveles del sistema (proceso, campo y estación), orientado a la integración de forma estándar de equipos de diferentes fabricantes, reduciendo la necesidad de utilizar convertidores de protocolo y minimizando los tiempos de ingeniería en dicha integración. Adicionalmente como parte de las necesidades del sector eléctrico, también contempla la utilización de sistemas de almacenamiento para diagnóstico, captación y procesamiento distribuido de la información, así como la optimización de costos tanto en el diseño como en su mantenimiento.

Al establecer la norma una comunicación a nivel de proceso y campo, permite un mayor control del sistema y una disminución considerable en el cableado eléctrico, enviando la información requerida en los IEDs por comunicaciones. La implementación debe ser cuidadosa, debido a que se tienen que seguir conservando las prestaciones operativas convencionales de los sistemas eléctricos clásicos como lo es tiempos de operación, seguridad, redundancia y confiabilidad. Una de las características del protocolo IEC 61850 que sirve de apoyo para cumplir con estas condiciones, es que ha sido diseñado para permitir actualizaciones tecnológicas en el área de las comunicaciones, debido a que está basado en TCP/IP e Ethernet.

La integración de los diferentes equipos electrónicos (IEDs), Gateway y otros dispositivos del sistema, se realiza en una arquitectura común mediante la utilización de la plataforma de comunicaciones denominada tecnología de red Ethernet (capas 1 y 2 del modelo OSI). Esta tecnología permite la conexión de dispositivos con distintas funciones y de diferentes fabricantes en una red común, sin embargo la IEC 61850 no define ninguna topología de red en particular, lo cual, implica un diseño robusto ante fallas y ataques informáticos para garantizar el correcto funcionamiento del sistema y aprovechar todos los beneficios de la norma e Ethernet, convirtiéndose el diseño de las comunicaciones en una parte fundamental en la implementación del protocolo.

El término topología se refiere a la forma en que está diseñada la red, bien sea física o lógicamente. Existen cinco tipos básicos: malla, estrella, árbol, bus y anillo [2]. Aunque las topologías básicas son ampliamente conocidas, es importante tener en cuenta algunas consideraciones al seleccionar alguna de ellas, debido a que cada sistema eléctrico de potencia posee su propia configuración y requerimientos particulares.

Dentro del alcance del artículo se pretende analizar y estudiar los elementos que se involucran en las redes Ethernet para sistemas de potencia eléctrica implementadas con la norma IEC 61850, planteando como objetivo presentar algunos criterios y consideraciones metodológicas y tecnológicas que se deben tener en cuenta en su diseño. Dado que un mal diseño de red puede generar pérdida de información, ocasionando una mala operación y en el peor de los casos hasta incendios y daño en los dispositivos conectados al sistema, sin contar con los millonarios costos que genera la 
pérdida del suministro de energía.

Para abordar el tema el documento está estructurado de tal manera que el lector tenga inicialmente una idea global de lo que es el protocolo IEC 61850 y los antecedentes a nivel mundial que se tienen. Continuando con un análisis más detallado del protocolo y definiendo los criterios de comunicación que se deben tener en cuenta en su diseño e implementación, con estas consideraciones definidas se realiza una prueba real con dispositivos analizando el comportamiento de la red y presentando las variables relevantes en ella.

\section{ANTECEDENTES Y ESTADO ACTUAL}

En los años noventa existían en Europa y USA desarrollos paralelos a nivel de estandarización de protocolos de comunicación para sistemas eléctricos. Mientras la IEC en Europa establecía una norma para las interfaces de los IEDs (se trataba de la norma T103 para la interfaz de comunicación de equipos de protección), en los Estados Unidos el electric power research institute (EPRI) trabajaba en un proyecto denominado "utility communications architecture" o UCA para desarrollar una infraestructura común de comunicaciones. El primer estándar establecido bajo el concepto de UCA en 1999 fue la norma IEC 60870-6 TASE.2 (ICCP) para la conexión abierta entre centros de control. En 1995 la IEC reconoció la necesidad de crear una norma general para las redes de comunicación en los sistemas de potencia eléctrica (subestaciones eléctricas inicialmente), y creó con ese fin varios grupos de trabajo, conformado por expertos de distintos países y con experiencias tanto en los protocolos IEC 60870 como con UCA. Al mismo tiempo que la IEC trabajaba en este proyecto, la EPRI, desarrollaba el UCA 2.0 para la definición de las comunicaciones dentro de la subestación. Sin embargo, la EPRI resolvió publicarlo como un "reporte técnico", para no competir en contra de los propósitos de la IEC y se acordó por parte de la IEC y la EPRI, el generar en conjunto una norma de aceptación mundial, la cual denominaron IEC 61850[1],
[25].

La norma IEC 61850, indudablemente despertó el interés de las áreas de protecciones y control de las empresas eléctricas, a partir de su publicación a fines de 2004 [3]. Inicialmente, los especialistas se concentraron en conocer los diversos aspectos que la componían, realizando proyectos piloto que permitieran conocer más detalladamente los aspectos prácticos de su aplicación en la automatización de subestaciones.

\subsection{Estado actual en Colombia}

En la actualidad, el marco regulatorio del mercado eléctrico Colombiano ha tenido mayor interés en satisfacer la demanda energética de todos los usuarios, con unos indicadores de calidad, seguridad y confiabilidad. Esto en parte debido, al actual mercado liberalizado del país y al auge mundial de optimizar los recursos energéticos coadyuvando al uso racional de energía. Lo anterior da origen a la necesidad de poder tener una supervisión y control del sistema eléctrico nacional.

De otro lado, el concepto de redes inteligentes (RI) está actualmente capturando la atención del sector eléctrico en todo el mundo y su implantación se está planeando o está en plena ejecución en especial en los países desarrollados. Una Colombia inteligente es una nación comprometida con un programa de energía sostenible, donde todos los sectores hagan uso eficiente de los recursos energéticos preservando el medio ambiente y logrando niveles adecuados de calidad [4]. En el informe anterior, también presentan dentro de los eventos más relevantes que han trabajado la temática de redes inteligentes en los últimos años en el país, la implementación y difusión de la norma IEC 61850 en el sector eléctrico.

En el sistema de transmisión nacional ya se cuenta con algunos sistemas eléctricos automatizados con el estándar IEC 61850; entre las más importantes se tienen los sistemas presentados en la tabla 1. 
Tabla 1. Sistemas eléctricos en Colombia con IEC 61850.

\begin{tabular}{|l|}
\hline S/E San Carlos $500 / 230 \mathrm{Kv}$ \\
\hline S/E Primavera $500 / 230 \mathrm{kV}$ \\
\hline S/E Copey $500 / 230 \mathrm{kV}$ \\
\hline S/E Porce $500 \mathrm{kV}$ \\
\hline S/E Bacata $500 / 230 \mathrm{kV}$ \\
\hline S/E Ocaña $500 / 230 \mathrm{kV}$ \\
\hline S/E Bolívar $500 / 230 \mathrm{kV}$ \\
\hline S/E Cerromatoso \\
\hline S/E La Reforma $230 \mathrm{kV}$ \\
\hline S/E Caño-Limón $230 \mathrm{kV}$ \\
\hline Central Hidroeléctrica Porce III \\
\hline Central Hidroeléctrica San Carlos \\
\hline S/E Mocoa $115 \mathrm{kV}$ \\
\hline
\end{tabular}

\subsection{Estado actual en el mundo}

La tendencia mundial hacia el uso racional de energía, optimización de recursos naturales y avances a nivel de comunicación, ha impulsado al desarrollo de tecnologías en todas las áreas del conocimiento entre ellas la implementación del protocolo IEC 61850 para sistemas eléctricos. Por otro lado es una propuesta que se puede extender al nuevo concepto denominado Smart Grid (red de distribución de energía eléctrica inteligente) que en los países desarrollados ya está en curso [28].

Dentro de la investigación realizada se encontró que se han implementado cerca de 400 subestaciones con protocolo IEC 61850 alrededor del mundo; entre las más importantes se tienen las presentadas en la tabla 2.

Tabla 2. Sistemas con protocolo IEC 61850 en el Mundo.

\begin{tabular}{|l|}
\hline S/E Nanqiao 500kV (China) \\
\hline S/E Polpaico 525kV (Chile) \\
\hline S/E Albrechtice 420/123/10kV (Grecia) \\
\hline S/E HaDong ECMS (Korea) \\
\hline S/E Zapata 230/115kV (México) \\
\hline S/E BeliyRast 750/500/110kV (Rusia) \\
\hline S/E Lawrence 230KV (US) \\
\hline S/E Dong Hoy 230kV (Vietnam) \\
\hline
\end{tabular}

\begin{tabular}{|l|}
\hline S/E Termozulia 230kV (Venezuela) \\
\hline S/E Chilca 500/220kV (Perú) \\
\hline S/E Pascuales 230/138/69kV (Ecuador) \\
\hline LNG-Lager Reganosa 132kV (Alemania) \\
\hline S/E ET Solalban 132kV (Argentina) \\
\hline S/E Aracuai (Brasil) \\
\hline H substation 400kV (Dubai) \\
\hline Chaingy 400/225/90kV (Francia) \\
\hline Substation Ranchi 400/220kV (India) \\
\hline
\end{tabular}

\section{ASPECTOS GENERALES DE LA IEC 61850}

IEC 61850 define dentro de su fundamento la existencia de tres niveles de operación en un sistema eléctrico (inicialmente subestaciones y extendido a centrales de generación hídricas en su edición 2) (Fig. 1).

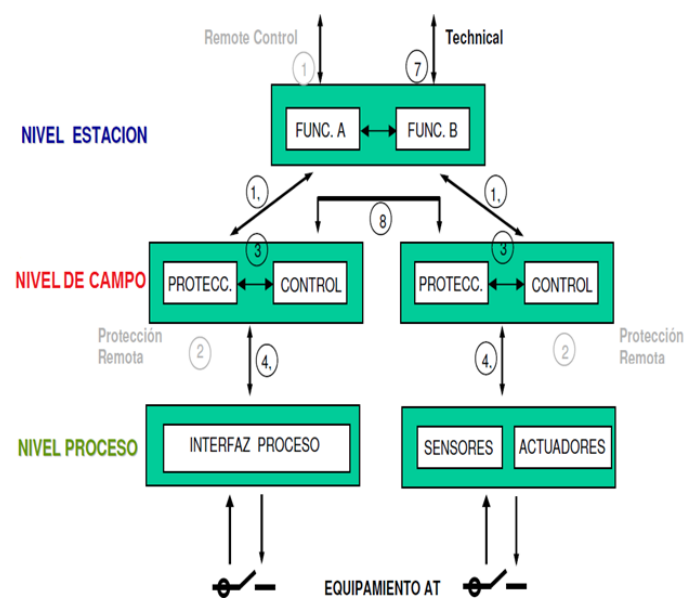

Fig. 1. Modelo de interfaz de un sistema de automatización de subestaciones (SAS) [10], [30].

Partiendo de esta premisa y ha manera general, la norma a partir de las funciones de control y protección de la subestación crea unos objetos o modelos de información, los cuales se interconectan entre sí mediante servicios de comunicación que luego son relacionados (mapeados) en la plataforma de protocolo de comunicación utilizada. Los datos son agrupados por restricciones funcionales (RF) organizados dentro de nodos lógicos (LN) en dispositivos lógicos (LD) ubicados en equipos físicos. En la Fig. 2 se presenta la organización de datos que plantea la norma. 


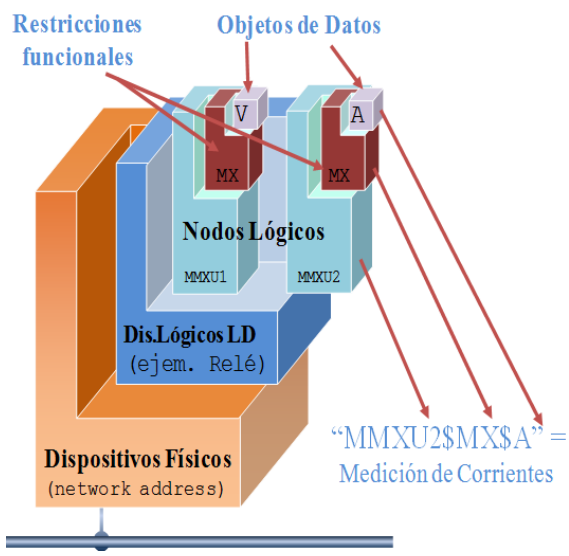

Fig. 2. Ejemplo de organización de información con IEC 61850 (elaboración propia basada en [10], [29]).

La estructura general de un dato en el protocolo IEC 61850 se presenta en la Fig. 3.

\section{BUK01/QOCSW1.Pos.ctIVal}

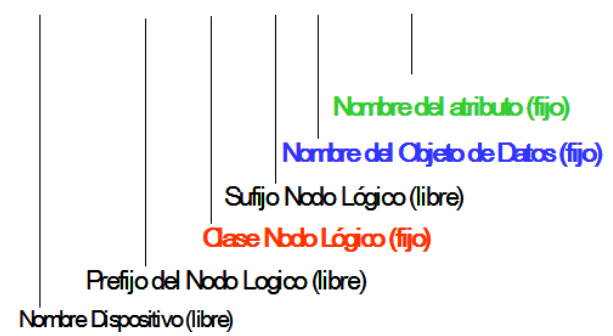

Fig. 3. Estructura general de un dato en el protocolo IEC 61850 (elaboración propia basado en [11], [12]).

La arquitectura para el intercambio de información en la IEC61850, se plantea en una estructura cliente/servidor. A nivel de la red de comunicaciones en sistemas eléctricos se pueden encontrar inicialmente tres grupos de información que fluyen en la estructura, los cuales dependiendo de avances tecnológicos podrían incrementar prestando mejores servicios en este tipo de aplicaciones [29]. El primero de ellos son los datos de operación, que hacen referencia a señales de alarmas, estados de equipos y señales de control del sistema eléctrico. Como segundo grupo se tienen los datos de procesos que involucran a nivel general, señales de medida y de disparo que tienen una mayor importancia a nivel eléctrico a causa de que con estos valores o señales se toman las decisiones en el sistema; como característica se tiene que requieren de una prioridad alta de flujo en la red. El tercer tipo que se puede encontrar en estas aplicaciones, son los datos de gestión y configuración del sistema, que básicamente es la gestión de dispositivos de la red y de equipos eléctricos, como la transferencia de archivos.

La transmisión de información implementando el protocolo IEC 61850 se puede realizar a través de servicios de comunicación que la misma norma establece (Fig. 4), entre los que se tienen la transferencia rápida de eventos (GOOSE), la sincronización de tiempo (NTP, IEE-1588), transferencia de archivos, mensajes MMS y gestión de Red (SNMP).

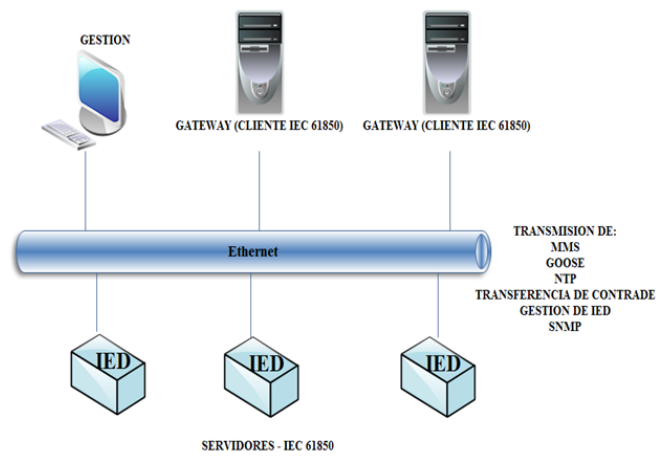

Fig. 4. Servicios de comunicación SNMP.

A nivel del modelo OSI la norma plantea el esquema presentado en la Fig. 5. La transmisión de información de operación se realiza a través de mensajes MMS, el cual es una especificación internacional y se puede definir como un sistema de transmisión de telegramas normalizado para la intercambio de datos en tiempo real, diseñado para alcanzar un gran nivel de interoperabilidad.

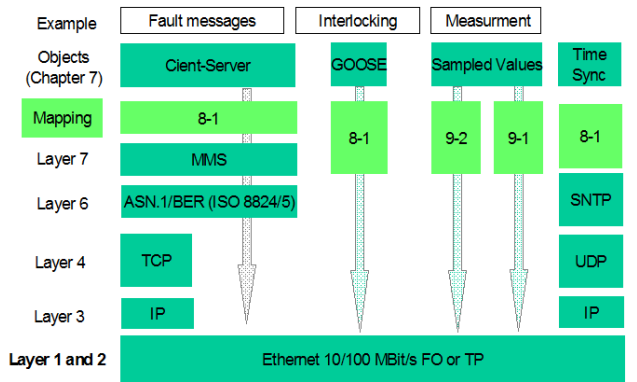

Fig. 5. Esquema propuesto por la norma IEC 61850a nivel del modelo OSI [13]. 
Los datos de procesos se transmiten a través de mensajes GOOSE o también nombrada como la transmisión rápida de eventos, la cual se basa en la norma IEEE 802.1q de Ethernet, con la posibilidad de enviar a través de la red mensajes con un bit de prioridad, para transmitir información que requiera de mucha velocidad, como es el caso de los disparos y eventos requeridos para enclavamientos en los sistemas eléctricos que oscilan desde los $150 \mathrm{~ms}$.

En la Fig. 6 se presenta el esquema de funcionamiento de la prioridad en los mensajes GOOSE; cuando es generado, al tener una prioridad mayor a las tramas comunes, este sobrepasa la cola de transmisión y el mensaje es enviado a la red, garantizando tiempos cortos en la transmisión en casos de tener avalancha de información.

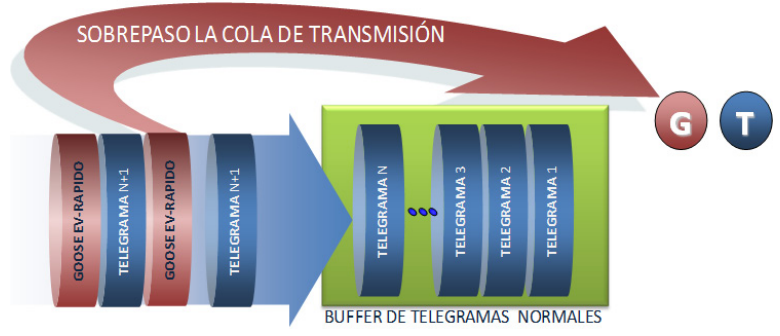

Fig. 6. Mensajes GOOSE con prioridad. (Elaboración propia basado en [13]).

Los mensajes del servicio GOOSE son de tipo multicast, es decir, que son transmitidos a varios dispositivos de la red, los cuales están suscritos para recibir el mensaje y posteriormente procesarlos. Estos datos son organizados en un DataSet.

Como la transmisión es de tipo multidifusión no existe un proceso de acuso de recibo del dato, por lo tanto, se requiere de un mecanismo de repetición del mensaje para asegurar la transmisión óptima del mismo. Esta retransmisión consiste en estar enviando repetidamente el mensaje GOOSE [26].

En estado estable, el envió se realiza cada cierto tiempo como se muestra en la Fig. 7, sin embargo cuando ocurre un evento nuevo (cambio de estado de la señal enviada), el intervalo de transmisión se acorta, y es progresivamente aumentado hasta llegar nuevamente al tiempo normal del ciclo en una relación según el ejemplo presentado en la Ec. 1.

$$
t_{S}=2^{N} \cdot S
$$

Con $\mathrm{N}=1,2 \ldots 3 \ldots$ para $\mathrm{t}_{\mathrm{s}}<\mathrm{Z}$ y $\mathrm{S}=5 \mathrm{~ms}$. Donde $\mathrm{Z}$ representa el intervalo de ciclo de monitoreo GOOSE.

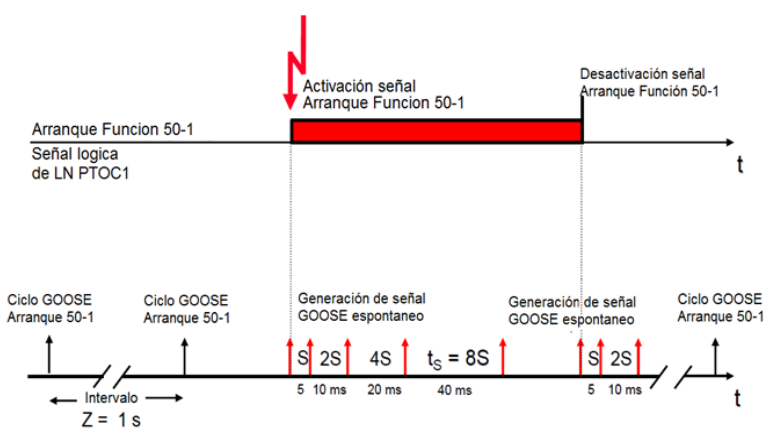

Fig. 7. Transmisión de mensajes GOOSE

(Elaboración propia basado en [13]).

El tiempo de transmisión es definido por [13] como se presenta en la Fig. 8. Donde el tiempo de transferencia de aplicación a aplicación (de F1 hasta F2), incluye el tiempo de procesamiento de envió realizada por el procesador de comunicación emisor DF1 (ta), el tiempo de retardo de la red Ethernet (tb) y el tiempo de procesamiento del receptor DF2 (tc). Dependiendo del fabricante el procesamiento del dispositivo varia aproximadamente entre 6 y $12 \mathrm{~ms}$, el tiempo de retardo de la red Ethernet varía de acuerdo al tráfico y topología [24]. En [10] se realiza el cálculo de este retardo a partir de una red LAN de 100MBits/s y varios esquemas típicos de subestaciones con un estimado del tráfico posible en cada caso, dando como resultado promedio un retardo máximo de 3,36ms. Obteniendo de lo anterior, que el tiempo de transmisión de un sistema en condiciones normales varia aproximadamente de 16 a 28ms, tiempo suficiente para el requerimiento de protección eléctrica, los cuales oscilan a partir de los $150 \mathrm{~ms}$ para operación de las funciones de protección. 


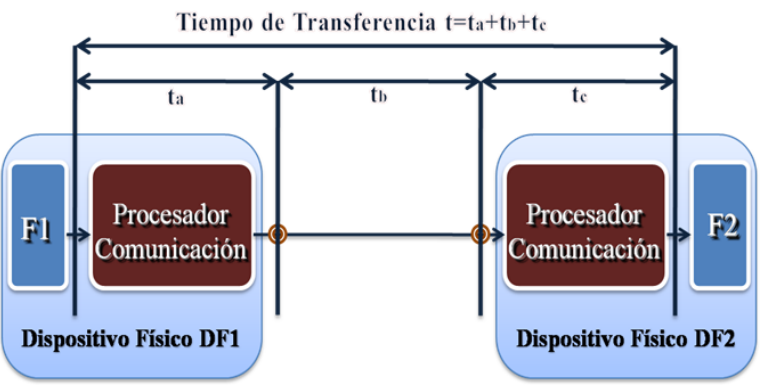

Fig. 8. Tiempo de Transmisión de datos.

(Elaboración propia basado en [13], [24]).

\section{A continuación se presenta una captura GOOSE obtenida con Wireshark:}

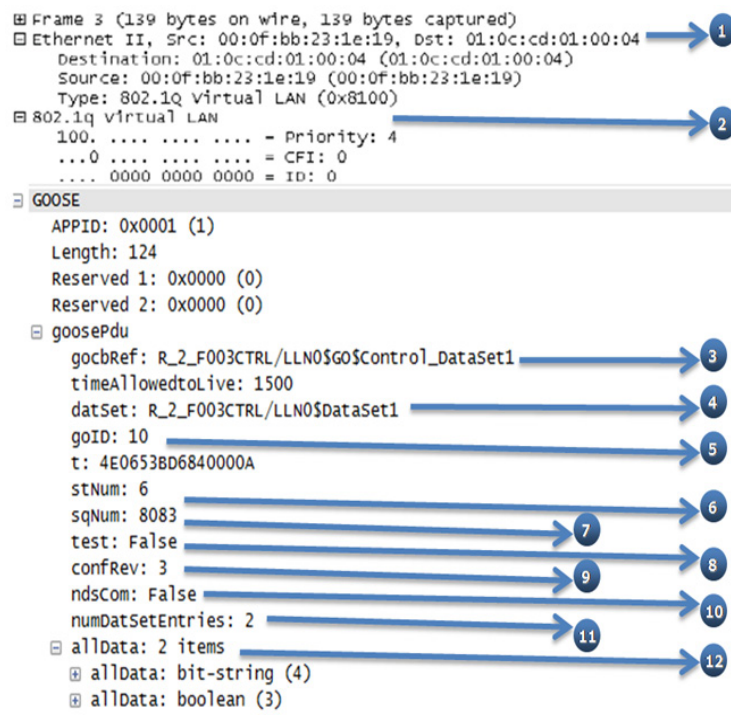

Fig. 9. Trama GOOSE.

La estructura mostrada en el gráfico anterior está compuesta por los campos mostrados en la tabla 3.
Tabla 3. Descripción campos de una trama GOOSE.

\begin{tabular}{|c|c|}
\hline 1 & $\begin{array}{l}\text { En (1) se presentalas direcciones MAC de la fuente } \\
\text { y destino del mensaje, estas son direcciones } \\
\text { virtuales generadas por cada IED en el momento } \\
\text { de la creación de un GOOSE, el rango es definido } \\
\text { en [13] y varía desde 01-0c-cd-00-00 hasta la } \\
01-0 \text { c-cd-01-ff. }\end{array}$ \\
\hline 2 & $\begin{array}{l}\text { En (2) se presenta los parámetros de la VLAN, } \\
\text { el primero de ellos es la prioridad que varía de } \\
1-7 \text { y que según las recomendaciones de [13], } \\
\text { para mensajes GOOSE no debe colocarse en un } \\
\text { valor menor a } 4 \text { (la norma define la prioridad en } \\
\text { 4). Otro de los parámetros es el ID, el cual indica } \\
\text { la VLAN que por defecto para estos mensajes } \\
\text { es 0, sin embargo, es posible utilizar otro ID. } \\
\text { Cuando se utilice una VLAN diferente de 0 para } \\
\text { este tipo de mensajes hay que tener en cuenta la } \\
\text { configuración de en los Switch debido a que el } \\
\text { GOOSE es generado con Tagged. }\end{array}$ \\
\hline 3 & $\begin{array}{l}\text { El gocbRef ( } 3 \text { ) indica la dirección donde se } \\
\text { encuentra el origen de la información contenida } \\
\text { en el mensaje. }\end{array}$ \\
\hline 4 & $\begin{array}{l}\text { En (4) se define el nombre del Dataset, el cual } \\
\text { se puede definir como el contenedor de la } \\
\text { información enviada desde el dispositivo fuente } \\
\text { a la red. }\end{array}$ \\
\hline 5 & $\begin{array}{l}\text { El goID (5) es un identificador que representa el } \\
\text { número ID de la aplicación de la trama Ethernet, } \\
\text { es asociado a la aplicación donde se configura los } \\
\text { mensajes GOOSE. }\end{array}$ \\
\hline 6 & $\begin{array}{l}\text { El stNum (6) es un número que incrementa } \\
\text { cuando existe un cambio de estado de algún dato } \\
\text { contenido en el Dataset. }\end{array}$ \\
\hline 7 & $\begin{array}{l}\text { El sqNum (7) es un parámetro que representa el } \\
\text { número de veces que ha sido enviado el mensaje } \\
\text { desde su último cambio de estado. }\end{array}$ \\
\hline 8 & $\begin{array}{l}\text { Test (8) indica si el GOOSE está en prueba, esta } \\
\text { función se utiliza en casos de que un IED este en } \\
\text { modo prueba. Al activar esta función los otros } \\
\text { IEDs detectan que el mensaje generado esta en } \\
\text { modo prueba y no realizan ninguna función. }\end{array}$ \\
\hline 9 & $\begin{array}{l}\text { ConfRev (9) representa la llave de compatibilidad, } \\
\text { es utilizado para tener un control de cambios y } \\
\text { garantizar que en la red circulen mensajes validos } \\
\text { para todos los integrantes del sistema. }\end{array}$ \\
\hline 10 & $\begin{array}{l}\text { El ndscom (10) indica si existe alguna falla o } \\
\text { incompatibilidad en la configuración. }\end{array}$ \\
\hline 11 & $\begin{array}{l}\text { En (11) numDataSetEntries indica la cantidad de } \\
\text { información que contiene el DataSet }\end{array}$ \\
\hline 12 & $\begin{array}{l}\text { En allData (12) se encuentra la información } \\
\text { compartida por el IED fuente. }\end{array}$ \\
\hline
\end{tabular}

\section{CRITERIOS Y CONSIDERACIONES DE DISEÑO DE RED}

El diseño de red de comunicación en sistemas 
eléctricos implementados con IEC 61850, toma importancia debido a lo primordial que se ha convertido el suministro de energía eléctrica en el mundo, convirtiéndose en uno de los más importantes servicios a nivel económico, político y social. En busca de brindar una mayor confiabilidad del sistema eléctrico, en esta sección se presentan algunos criterios y consideraciones de diseño para que se tengan en cuenta en el desarrollo de proyectos implementados con la norma IEC 61850.

\subsection{Cálculo de disponibilidad del sistema}

En los esquemas convencionales de medida, control y protección de sistemas de potencia eléctrica se dispone de una serie de dispositivos independientes entre sí. Con la estructura convencional de control es posible calcular una disponibilidad de estos elementos para operar realizando las funciones esperadas. Teniendo en cuenta que este factor puede afectar la operación de la parte eléctrica; es importante determinar si la introducción de arquitecturas basadas en redes de dispositivos con equipos configurados en una red local tipo LAN, mejoran o por el contrario impactan negativamente la disponibilidad de las subestaciones y centrales eléctricas [5], [22].

Por definición la disponibilidad de un equipo está definida como la relación entre el tiempo durante el cual el sistema está en estado normal de funcionamiento con respecto al tiempo total de operación [5]. Por lo cual se puede decir que es el valor que expresa la probabilidad que el sistema o función trabaje según lo especificado. Para el cálculo de disponibilidad se tienen en cuenta las siguientes características las cuales influyen directamente en su valor (para más detalles acerca de este cálculo ver [5], [22]).

- Rata de falla: Expresa la probabilidad de que un componente del sistema vaya a fallar después de un tiempo t. Esta característica presenta tres períodos principales en la vida de un componente: mortalidad prematura, período de operación útil, período de decadencia.

- Tiempo medio para fallar (MTTF): Es igual al tiempo estimado de vida de un componente en el cual este no presenta fallos. Es igual al inverso de la rata constante de fallas en el ciclo de vida útil de un equipo (Ec. 2).

$$
M T T F=\frac{1}{\lambda}
$$

- Tiempo medio de reparación (MTTR): es el tiempo desde que sucede un error hasta que el sistema funciona otra vez correctamente. Esta variable tiene dos componentes principales: el tiempo de reacción ante el reporte de una falla y el tiempo de reparación en sitio del equipo.

- Tiempo medio entre fallas (MTBF): está definido por el valor medio entre dos fallas sucesivas y está dado por la suma del tiempo medio para fallar y el tiempo medio de reparación (Ec. 3).

$$
M T B F=M T T F+M T T R
$$

A partir de las características enunciadas anteriormente se define que la disponibilidad (D) está dada por la Ec. 4.

$$
\begin{gathered}
D=\frac{M T T F}{M T B F} \\
D=\frac{M T T F}{M T T F+M T T R} \\
D=\frac{1}{1+\lambda \cdot M T T R}
\end{gathered}
$$

Adicionalmentepara el cálculodedisponibilidad a nivel de sistemas de control basados en la norma IEC 61850 se pueden tener dos clases de estructuras:

- Estructura paralela: Se dice que dos componentes o más de un sistema están en paralelo cuando solo uno de éstos es necesario para su correcto funcionamiento. Para dos componentes en paralelo con disponibilidades Dm y Dn, el valor de esta variable conjunta está definida por la Ec. 5 .

$$
D s=D m+D n-D m \cdot D n
$$

- Estructuras en serie: Se dice que dos 
componentes o más de un sistema están en serie, cuando todos estos elementos deben estar operativamente buenos para su correcto funcionamiento. La disponibilidad resultante está definida por la Ec. 6.

$$
D_{S}=\prod_{i=1}^{n} D_{i}
$$

Donde $\mathrm{n}$ es el número de componentes con disponibilidad en serie.

\subsection{Desempeño de la red Ethernet}

El desempeño de una red de comunicaciones está determinado por el tiempo que demora un mensaje desde su origen hasta llegar a su destino. Esta demora se denomina latencia. Para la medición de dicha variable de red se debe tener en cuenta los siguientes datos [6]:

- $\mathrm{V}_{\mathrm{TX}}$ : Velocidad de transmisión de los puertos.

- Jitter de los equipos de red: Representa el tiempo que demora el dispositivo en leer un mensaje entrante y decidir su destino.

- Saltos: Es la cantidad de dispositivos por los que debe pasar el mensaje desde el origen hasta el destino.

- $\mathrm{T}_{\text {M-paquete }}$ (tamaño máximo de un paquete Ethernet): 1530 Byte (30 Byte son datos del encabezado y secuencia de chequeo del mensaje).

Por lo tanto, el tiempo de transmisión del paquete entre dos nodos de la red contiguos esta dado por la Ec. 7.

$$
T_{p}=T_{M-\text { paquete }}\left(\frac{1}{V_{T X}}\right)
$$

Y el tiempo de latencia del mensaje $\left(\mathrm{T}_{\mathrm{M}}\right)$ para pasar de un switch o dispositivo a otro está dada por la Ec. 8.

$$
T_{m}=\left(T_{p} \cdot c_{1}+T_{l}\right)+\left(T_{p} \cdot c_{2}+T_{l}\right)
$$

Donde $c_{1} \quad y_{2} c_{2}$ son el puesto en la cola del mensaje en el switch 1 y 2 respectivamente y $T_{l}$ es el tiempo de latencia de un conmutador que dependiendo del fabricante varía de 5 a $10 \mu \mathrm{s}$. Con base en lo anterior, se puede deducir que la expresión para calcular la latencia de la red a través de $\mathrm{N}$ switch es mediante la Ec. 9.

$$
\begin{aligned}
& T_{m}=\sum_{i=1}^{N}\left(T_{p} \cdot c_{i}+T_{l}\right) \\
& T_{m}=N \cdot T_{l}+T_{p} \cdot \sum_{i=1}^{N} c_{i}
\end{aligned}
$$

De acuerdo a lo anterior, se tiene que el tiempo de transmisión del paquete entre dos puertos adyacentes de la red, para un esquema típico de sistemas con IEC 61850, conociendo que estos esquemas son implementados con una velocidad de transmisión de $100 \mathrm{Mbps}$, es:

$$
\begin{aligned}
& T_{p}=1530 \text { Byte }\left(8 \frac{\text { Bits }}{\text { Byte }}\right)\left(\frac{1}{100 M b p s}\right) \\
& T_{p}=122,4 \mu s
\end{aligned}
$$

El tiempo de latencia de un mensaje para pasar de un dispositivo de red a otro, es de:

$T_{m}=(122,4 \mu s \cdot 2+7 \mu s)+(122,4 \mu s \cdot 4+7 \mu s)$

$T_{m}=748,4 \mu s$

Para más detalles acerca del cálculo del desempeño de la red remítase a [6] [15].

\subsection{Topología de la red Ethernet}

Como se expuso en la sección 1, la norma IEC 61850 no define ninguna topología de red en particular, lo que implica la realización de un diseño robusto ante fallas para garantizar el correcto funcionamiento del sistema. Como se ha mencionado hasta el momento la especificación tiene su fundamento en Ethernet, por lo cual se hace necesario basarse en protocolos $y$ algoritmos que brinden robustez y confiabilidad orientadas hacia este nivel. En este apartado se estudiarán tres de los protocolos que pueden ser implementados en este tipo de red. Para comenzar, a nivel de redes de comunicaciones basadas en IEC 61850 se puede encontrar e implementar los siguientes tipos de configuración [22]: 


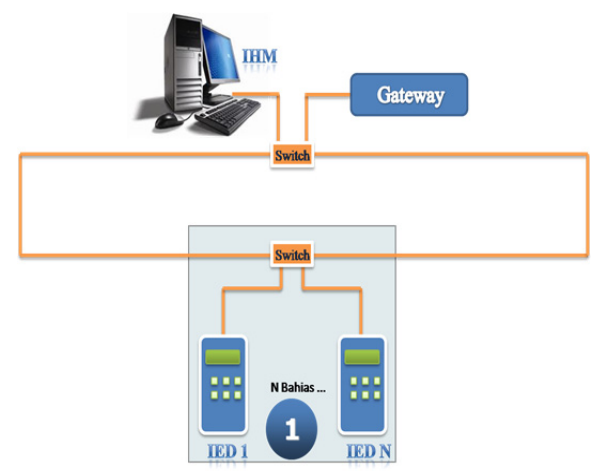

Fig. 10. Topologías de red en estrella.

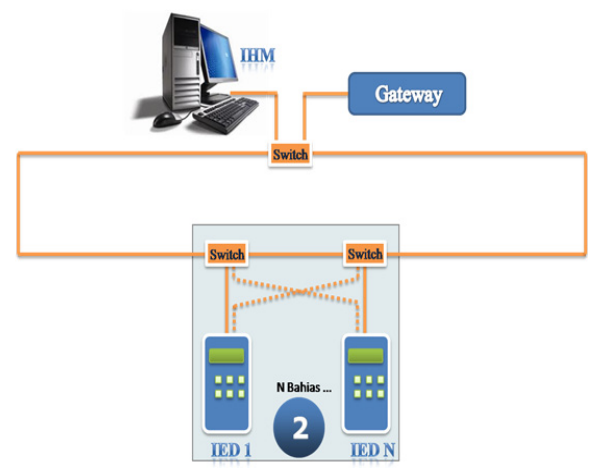

Fig. 11. Topologías de red en estrella redundante. (Elaboración propia basado en [2], [3], [22])

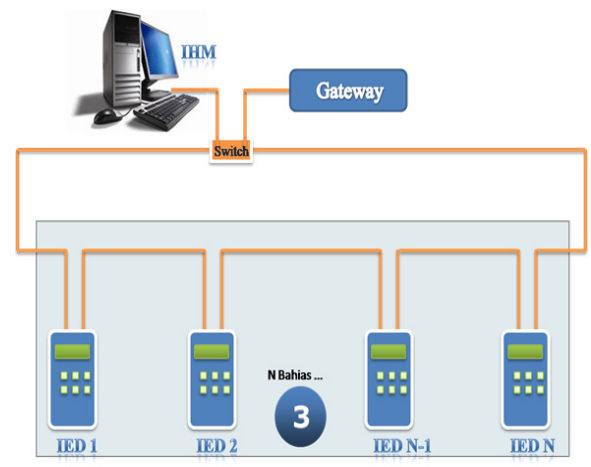

Fig. 12. Topologías de red en anillo. (Elaboración propia basado en [2],[3],[22])

En las Fig. 10, 11 y 12, se presentan las topologías típicas de red utilizadas en sistemas que implementan protocolo IEC61850. En la Fig. 10 se muestra un tipo de estructura llamada en estrella o radial; se caracteriza por tener un enlace físico uno a uno y es generalmente usado en aplicaciones pequeñas, no posee redundancia a nivel de comunicación. La conexión de la Fig. 11 es un esquema de red redundante en estrella, el cual consta de dos enlaces físicos que van a dispositivos de red diferentes, este tipo de diseño dependiendo del protocolo de redundancia puede transmitir simultáneamente (protocolo PRP) o puede funcionar en modo principal-respaldo, en el que solo un puerto transmite en un tiempo $t$ determinado. La tercera estructura enunciada en la Fig. 12, es una configuración en anillo en el que los IEDs se comportan como dispositivos de red, los protocolos de redundancia utilizados en este tipo de topologías son el RSTP y HSR-PRP.

Es posible tener esquemas mixtos en las que se combinen las configuraciones nombradas anteriormente, pero implica tener un poco de precaución en el diseño y programación de la red [15]. Adicionalmente a los protocolos de comunicación necesarios para la implementación de una configuración determinada, los switch, router y otros equipos de comunicación que se deseen utilizar en un sistema de potencia eléctrica, se establece unos requerimiento mínimos a nivel de condiciones ambientales, mecánicas y eléctricas, las cuales se especifican en [14].

A continuación se describe de forma general los tres protocolos de redundancia a nivel de capa 2, que pueden ser utilizados en sistemas donde se implemente el protocolo IEC61850.

\subsubsection{IEEE 802.1w RSTP (rapid spanning tree protocol)}

La función principal de los switch Ethernet consiste en remitir mensajes entre sus puertos. El lee el mensaje que entra por un puerto y determina la dirección MAC del equipo destino de dicho mensaje, remitiéndolo al puerto donde se encuentra el equipo con la MAC respectiva. Estos dispositivos trabajan a niveldecapa dos del modelo OSI y generalizando su funcionamiento, se basan en la creación dinámica de una base de datos con las direcciones MAC asociadas a cada puerto. Cuando llega un mensaje que posee una dirección MAC que el switch no conoce, este retransmite el mensaje por todos sus puertos y espera la respuesta del MAC respectivo para actualizar su base de datos de direcciones, lo 
cual es realizado a través del protocolo ARP. Si la topología de red es en configuración anillo, los mensajes podrían quedarse dando vueltas indefinidamente en la red, lo que podría saturar el ancho de banda hasta que ésta se bloqueara. El mecanismo definido por la norma IEEE 802.1w RSTP previene estos sucesos reconfigurando lógicamente la red [7].

RSTP fundamentalmente lo que realiza es una partición lógica de los anillos de tal manera que se formen redes tipo cascada donde no haya loops de tráfico de información. Si presenta una falla, el protocolo reconfigura automáticamente la red y en el orden de milisegundos forma una nueva topología que incluye nuevamente todos los equipos de la red. El protocolo RSTP utiliza las siguientes pautas y procedimientos para su convergencia [8]:

- Los switch poseen la capacidad de transmitir entre sí mensajes de configuración denominados BPDU.

- Durante el diseño y cuando se ejecuta el protocolo se selecciona un switch dentro de toda la red como principal (Root - con la menor prioridad asignada), los otros dispositivos restantes de la red toman el rol de designados. Ante la falla del equipo principal, el rol es asumido por el switch con la segunda menor prioridad, y así sucesivamente. A partir de lo anterior se fortalece el concepto del buen diseño de la topología, orientándose en este punto a la selección correcta de las prioridades.

- Todos los witch calculan y almacenan en una base de datos la dirección y costo de transmitir mensajes hasta el dispositivo principal, con el fin de determinar varios caminos y los costos asociados a cada uno de ellos.

- Cada switch configura a partir de la base de datos mencionada anteriormente, cada uno de sus puertos con un estado específico los cuales pueden ser: puerto descartado, puerto en aprendizaje, puerto en transmisión, así como también su rol dentro de la red (principal, designado, bloqueado).

- El switch determina si el puerto va a participar activamente en la red, si es el caso, lo activa en modo "aprendizaje" durante un lapso entre 6 y 40s, donde el puerto aun cuando no participa en el tráfico de la red, escucha los mensajes de configuración "BPDU" y actualiza su base de datos con la información de direcciones y costos de los enlaces. Luego del proceso de aprendizaje el puerto es activado en modo transmisión y puede ser identificado con alguno de los roles en el tráfico de datos, esto es como principal, bloqueado o designado.

- Al ocurrir una falla de algún enlace, el dispositivo con el mejor nuevo camino le "propone" al equipo adyacente que identifique su puerto del segmento como puerto principal. Si el switch adyacente está de acuerdo con la propuesta, modifica el rol del puerto y envía un mensaje de aceptación. Este proceso se realiza en toda la red hasta la nueva convergencia del protocolo, definiendo asíla nueva topología.

\subsection{2. $P R P$}

El protocolo de redundancia paralela es un nuevo concepto a nivel de configuración de redes Ethernet y es propuesto como alternativa de configuración en la norma IEC 61850 segunda edición. La base principal de PRP es el uso de dos redes Ethernet independientes para la transmisión de datos; la trama es replicada por el nodo de envió y es trasmitida a ambas redes de forma simultánea (Fig. 13). En el receptor es aceptada la primera trama que recibe y descartada la segunda, este proceso se realiza a través de un administrador de redundancia el cual permite enviar solo una trama a los niveles superiores de su pila de comunicación [17].

La forma en que es posible descartar la trama duplicada en el receptor es con la adición de cuatro octetos dentro de ella, esta información es denominada redundancy control trailer (RCT) y contiene los siguientes parámetros:

- 16 bit para el número de secuencia: Es la identificación de la trama en el receptor, de esta manera se conocen las tramas duplicadas en el nodo receptor. 
- 4 bit para el identificador de LAN, en el ejemplo de la Fig. 13. Red A o red B.

- 12 bit para el tamaño de la trama.

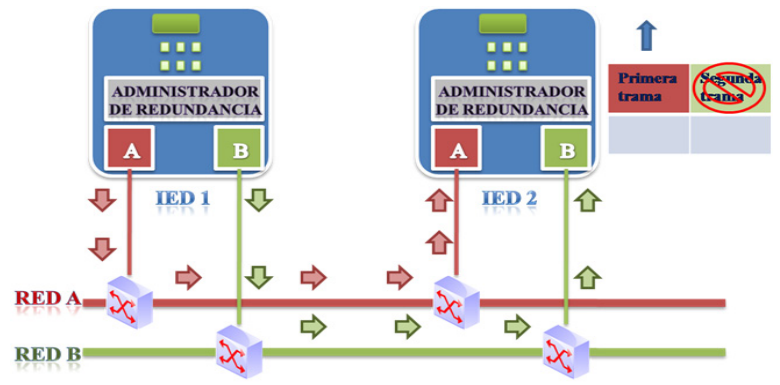

Fig. 13. Configuración PRP

(Elaboración propia basado [16][18])

El protocolo al tener una estructura paralela proporciona una redundancia confiable, en caso de tener una falla en la red A, la red B transmite la información que a causa de la falla se dejó de transmitir en A. Sin embargo este protocolo tiene una falencia a nivel de fallas múltiples en las dos redes. Por ejemplo, si falla la red A del IED 1 y la red B del IED 2, aunque la red $\mathrm{B}$ del IED 1 envíe la trama hacia el IED 2 esta información se perderá debido a la simultaneidad de la falla en las dos redes. Como solución a este problema se contempla la propuesta de utilizar HSR-PRP, como se presenta en el siguiente apartado.

El PRP se encuentra actualmente en desarrollo por parte de los fabricantes y existen algunos proyectos pilotos para su implementación, este protocolo ha tenido una evolución lenta ocasionada a que depende mucho del hardware que se, lo cual ha generado un desarrollo tecnológico más profundo en este sentido. Para equipos que no soporten PRP y se necesiten integrar a una topología de este estilo, se contempla el creación de lo que denominan RedBox, el cual conecta el dispositivo radialmente y este su vez es conectado a la red PRP.

\subsubsection{PRP/HSR (parallel redundancy protocol- high available seamless redundancy principle)}

Es una propuesta realizada por algunos fabricantes e incluida en la norma IEC 62439-
3 en pro al mejoramiento de PRP con respecto a fallas múltiples en la red. Funciona con los principios de PRP pero a diferencia de este, PRP/ HSR utiliza una red común en configuración anillo y con estructura paralela. Este protocolo utiliza el RCT para posibilitar el descarte de tramas duplicadas. En la Fig. 14 se presenta un ejemplo de esta configuración, básicamente la tecnología consiste en la generación de dos tramas con diferente red (red A, red B) en el nodo emisor el cual transmite la información por cada uno de sus puertos. Las dos tramas llegan al receptor, sin embargo a pesar de usar el mismo medio físico, al tener redes diferentes no existe colisiones. Con la ayuda del administrador de redundancia se acepta la primera trama y se descarta la segunda evitando información repetida en los niveles superiores de la pila de comunicación.

De manera similar a PRP este tipo de estructuras depende mucho del hardware y actualmente se encuentra en desarrollo por los fabricantes.

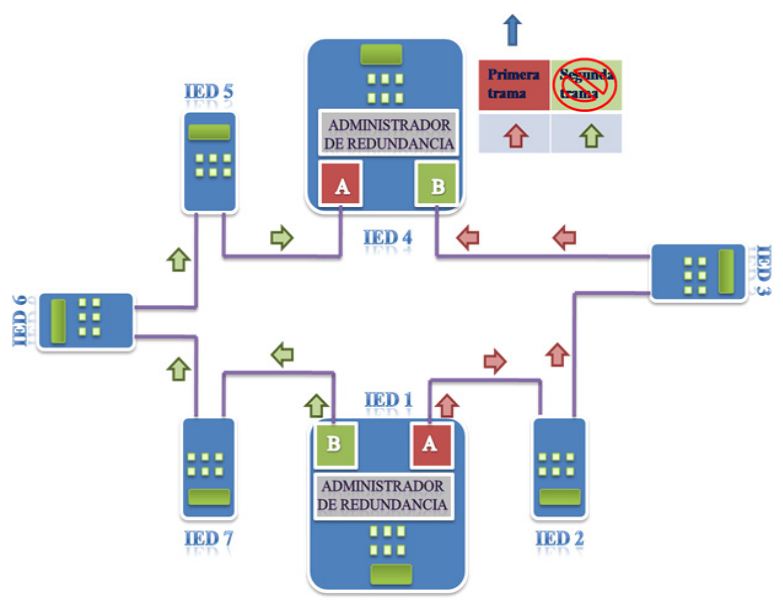

Fig. 14. Configuración PRP.

(Elaboración propia basado [17][18]).

\section{DIFERENCIAS DE IEC 61850 CON OTRAS TECNOLOGÍAS}

La norma IEC 61850 más que ser un protocolo de comunicación, es un estándar de diseño para redes eléctricas. Aunque la finalidad de la norma es el poder centralizar, supervisar y controlar sistemas de potencia eléctrica, la principal diferencia con otras tecnologías es 
que su estructura es orientada y diseñada para estandarizar ingeniería, optimizar procesos de diseño y garantizar interoperabilidad entre los equipos integrados al sistema [32].

Los protocolos utilizados en aplicaciones similares han sido desarrollados por los propios fabricantes de los IEDs, lo que hace de este desarrollo un uso exclusivo. Entre estas tecnologías se encuentran el courier (MICOM), SPA (ABB), profibus (SIEMENS), SEL ASCII (SEL) entre otros.

Existen otras técnicas que se han desarrollado en pro de generar sistemas de comunicaciones abiertos y garantizar una homogeneidad entre los diferentes marcas de IED's, este trabajo fue consecuencia de las dificultades que trajo el tener diversos dispositivos de diferentes fabricantes en un mismo sistema y la particularidad técnica que esta generaba. Cada modificación, mejora o integración necesitaba el uso exclusivo de personal especializado en cada tecnología, lo que acarreaba costos elevados tanto en el desarrollo del proyecto como en su mantenimiento. Dentro de los estándares de sistemas abiertos de comunicación se encuentran MODBUS (serial), DNP 3.0 (serial, TCP/IP), IEC 60870 (serial, TCP/IP) ente otros; y aunque lograron un buen avance en interoperabilidad, no cumplen totalmente con el hecho de ser abiertos debido a que cada fabricante interpretaba el protocolo de cierta manera. En parte, este fenómeno se dio por el diseño propio de cada uno de ellos, es decir que estaban orientados a la transmisión de información casi de cualquier tipo, más no a un tipo de flujo de datos con características particulares, como lo es el que se usa en sistemas de potencia eléctrica. Una de las mayores diferencias y beneficios del protocolo IEC 61850 es precisamente que se modelo para sistemas de potencia eléctrica, teniendo en cuenta cada uno de los elementos que se involucran en el sistema y logrando su modelamiento en UML lo que garantiza documentación y escalabilidad.

Al tener una estandarización en la ingeniería se minimizan los tiempos en el desarrollo de los proyectos tanto en el diseño como en el comisionamiento, de la misma manera se reducirán tiempos en mantenimiento y en consignaciones. Todo lo anterior impactando directamente en los costos del proyecto y costos operativos, lo que es finalmente el objetivo principal de las empresas del sector eléctrico [32].

\section{ESCENARIO DE PRUEBA COMPORTAMIENTO EN EL TIEMPO}

Como prueba al desempeño del sistema se realiza un montaje de equipos, en el cual se incluyen 16 bahías de una subestación. En la Fig. 15 se presenta el esquema de prueba realizado para el análisis, consta de 13 bahías de línea, 2 bahías de transformación y un acople de barras, las cuales en promedio cada una contiene 70 señales para supervisión control y protección. La topología de red implementada es en anillo como se muestra en la Fig. 12. Se utilizó un gateway para la supervisión de mensajes MMS y wireshark para analizar el tráfico de la red tanto de mensajes MMS como el flujo de mensajes GOOSE.

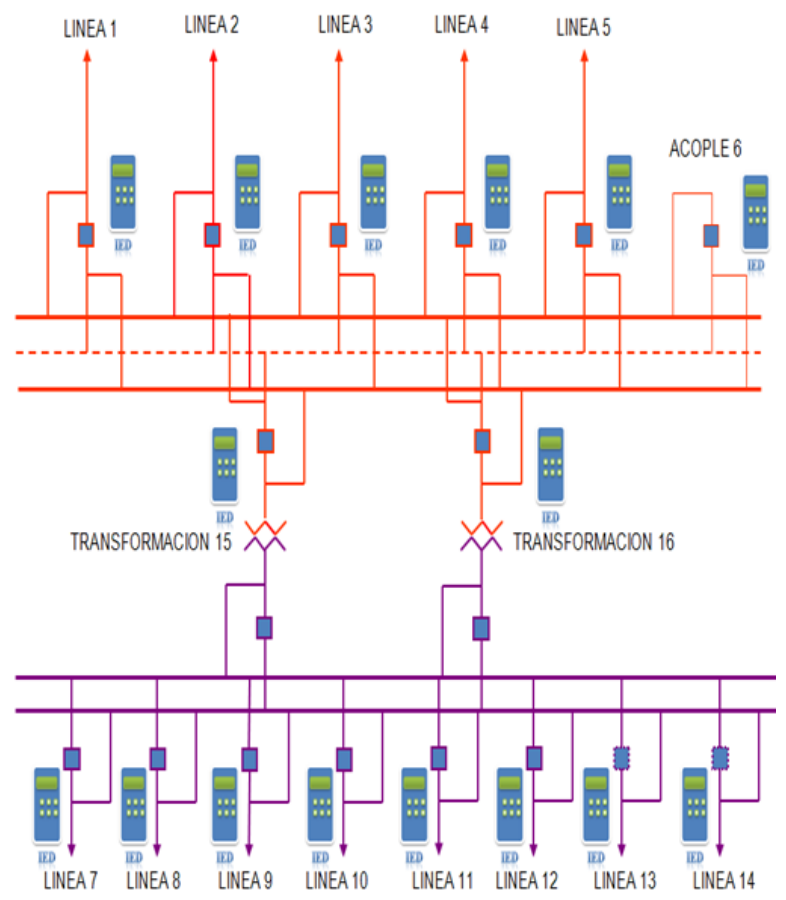

Fig. 15. Unifilar subestación eléctrica con 16 Bahías. 
La Fig. 16 presenta el tipo de flujo de datos en estado normal donde el tiempo de muestreo fue de 1 hora. En esta se observa que el mayor tráfico en la red es de tipo GOOSE con un $38,72 \%$, seguido por los tipos internet protocol (IP) en el cual los mensajes MMS tienen alrededor de un 19.40\%; esto refleja que las variables contenidas en un esquema como este, no presentan cambios significativos en relación al tiempo, esto se afirma conociendo que los mensajes MMS son enviados espontáneamente cuando ocurre un cambio de valor en alguna variable del reporte.

\begin{tabular}{|c|c|c|c|c|}
\hline \multirow[b]{2}{*}{ Probol } & \multicolumn{2}{|c|}{ Display fiter: nome } & \multirow[b]{2}{*}{ Ybives } & \multirow[b]{2}{*}{ Bytes } \\
\hline & Wpadets & Padeles \% & & \\
\hline$\nabla$ Frame & $100,0 \%$ & 6526 & $100,0 \%$ & 725556 \\
\hline$\nabla$ Etternet & $100,0 \%$ & 6526 & $10,0 \%$ & 725556 \\
\hline coose & $58,2 \%$ & 2527 & $51,00 \%$ & 360981 \\
\hline Adtress Resolition Frotocol & $2,93 \%$ & 191\lceil & $1,58 \%$ & 11460 \\
\hline$\nabla$ internet Probool & $56,83 \%$ & 300 & $4 6 \longdiv { 0 8 \% }$ & 333379 \\
\hline$\nabla$ Trassmisision Control Probool & $47,5 \%$ & 3129 & $37,91 \%$ & 274334 \\
\hline$\nabla$ TRT-150 on TOP-PFC1006 & $19,40 \%$ & 1266 & $21,71 \%$ & 157053 \\
\hline 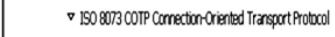 & $19,40 \%$ & 1266 & $21,71 \%$ & 15703 \\
\hline$\nabla 1508227-1051$ Session frotocol & $19,40 \%$ & 1266 & $21,71 \%$ & 157053 \\
\hline$\nabla$ ISO $8327-1051$ Session Probol & $19,40 \%$ & 1266 & $21,71 \%$ & 157053 \\
\hline$\checkmark 1500823$ OSI Presentation Probool & $19,40 \%$ & 1266 & $21,71 \%$ & 157053 \\
\hline ISO/EC C506 MSS & $19,40 \%$ & 1266 & $21,71 \%$ & 157053 \\
\hline Oata & $2,36 \%$ & 154 & $1,96 \%$ & 14181 \\
\hline$\nabla$ user Dabayam Protocol & $8,00 \%$ & 574 & $8,11 \%$ & 50685 \\
\hline Simple Netwodk Mrangement Protbol & $8,00 \%$ & 522 & $7,27 \%$ & 52509 \\
\hline Nebios Name Service & $0,0 \%$ & $46 \Gamma$ & $0,58 \%$ & 4228 \\
\hline Data & $0,09 \%$ & $6 \Gamma$ & $0,26 \%$ & 1864 \\
\hline Internet Cortrol Messoge frotcol & $0,09 \%$ & $6 \Gamma$ & $0,05 \%$ & 300 \\
\hline 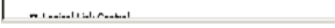 & inem & $\mathrm{m} \Gamma$ & neren & .m \\
\hline
\end{tabular}

Fig. 16. Tráfico de red en estado normal de una subestación eléctrica con 16 bahías.

Otro factor que se analiza es el comportamiento de la información en el momento en que ocurre un evento en la subestación; para ello se simulo una falla eléctrica en dos de sus bahías operando la función de sobrecorriente en ambos casos. En la Fig. 17 se presenta el comportamiento del tráfico en la red durante el fenómeno capturado con wireshark.

La línea de color rojo representa el tráfico de GOOSE, como se observa, su comportamiento es constante durante toda la captura y evidencia que en condiciones normales, existe mayor tráfico de GOOSE que de mensajes MMS la cual es dibujada de color azul. La línea negra es el flujo total en la red y cuyas componentes más significativas son los dos tipos de información enunciados anteriormente. Durante la ocurrencia de un evento común en el sistema se puede observar como incrementa el tráfico de mensajes MMS y como el GOOSE conserva su tendencia. Después del evento se presenta una estabilidad de los datos tipo MMS y se puede ver que aunque la falla esta en el orden de 500 a $1000 \mathrm{~ms}$, el comportamiento de la red ante el evento está alrededor de los $5 \mathrm{~s}$, lo que da origen al estudio del comportamiento del sistema en condiciones extremas de tráfico, para lo cual se generaron las pruebas que se presentan a continuación.

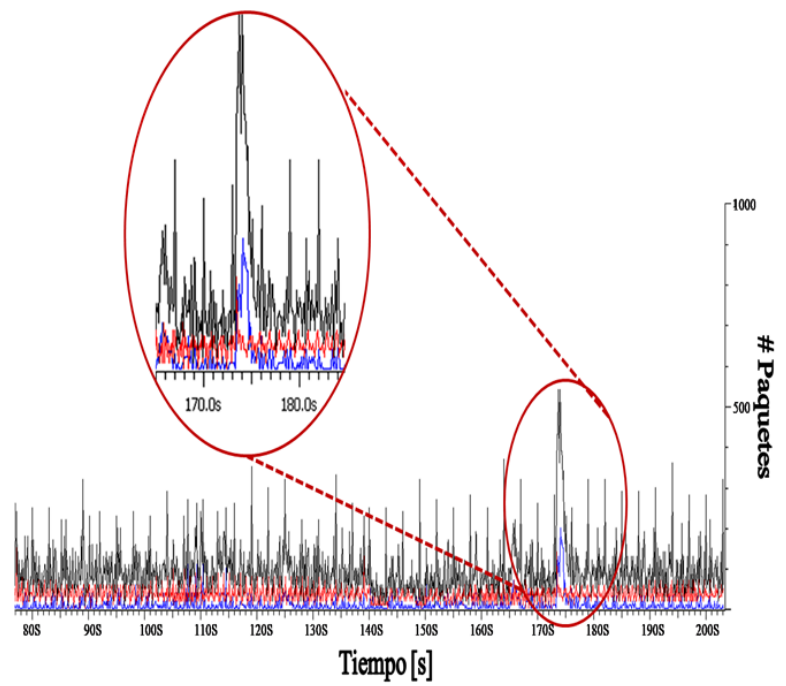

Fig. 17. Tráfico de red en presencia de eventos de una subestación eléctrica con 16 bahías.

Para observar el comportamiento del sistema en caso de avalancha, se realizó simultáneamente un ping a toda la red con diferencias de $4 \mathrm{~ms}$ entre cada pregunta, dando como resultado los datos presentados en la Fig. 18. Durante la prueba ocurrió un evento en el cual se perdieron 5 paquetes con un tiempo de recuperación de 88 y $10 \mathrm{~ms}$ en los dos equipos que fallaron. Sin embargo ninguno de los eventos que se generaron en la subestación se perdió y no excedieron la resolución de 3ms. 


\begin{tabular}{|c|c|c|c|c|c|c|}
\hline \multicolumn{2}{|c|}{ Enviados } & \multicolumn{3}{|c|}{ Recibidos } & \multicolumn{2}{|c|}{ Perdidas } \\
\hline \multicolumn{2}{|c|}{130167} & \multicolumn{3}{|c|}{130163} & \multicolumn{2}{|c|}{5} \\
\hline \multicolumn{7}{|c|}{ Tiempo recuperacion del sistema (ms): } \\
\hline \multicolumn{2}{|c|}{ Minimo } & \multicolumn{3}{|c|}{ Maximo } & \multicolumn{2}{|c|}{ Promedio } \\
\hline \multicolumn{2}{|c|}{88} & \multicolumn{3}{|c|}{88} & \multicolumn{2}{|c|}{88} \\
\hline \multicolumn{7}{|l|}{ Dispositivos: } \\
\hline \multirow[t]{2}{*}{ Direccion IP } & \multicolumn{3}{|c|}{ Ping } & \multicolumn{3}{|c|}{ Recovery Time (ms) } \\
\hline & Enviados & Recibidos & Perdidos & Minimo & Maximo & Promedio \\
\hline 10.122 .84 .31 & 8159 & 8156 & 3 & 88 & 88 & 88 \\
\hline 10.122 .84 .32 & 8142 & 8142 & 0 & 0 & 0 & 0 \\
\hline 10.122 .84 .33 & 8142 & 8142 & 0 & 0 & 0 & 0 \\
\hline 10.122 .84 .34 & 8142 & 8142 & 0 & 0 & 0 & 0 \\
\hline 10.122 .84 .36 & 8141 & 8141 & 0 & 0 & 0 & 0 \\
\hline 10.122 .84 .37 & 8141 & 8140 & 0 & 0 & 0 & 0 \\
\hline 10.122 .84 .38 & 8139 & 8139 & 0 & 0 & 0 & 0 \\
\hline 10.122 .84 .40 & 8138 & 8138 & 0 & 0 & 0 & 0 \\
\hline 10.122 .84 .41 & 8137 & 8137 & 0 & 0 & 0 & 0 \\
\hline 10.122 .84 .42 & 8134 & 8134 & 0 & 0 & 0 & 0 \\
\hline 10.122 .84 .43 & 8133 & 8133 & 0 & 0 & 0 & 0 \\
\hline 10.122 .84 .112 & 8131 & 8131 & 0 & 0 & 0 & 0 \\
\hline 10.122 .84 .114 & 8128 & 8128 & 0 & 0 & 0 & 0 \\
\hline 10.122 .84 .119 & 8125 & 8122 & 2 & 10 & 10 & 10 \\
\hline 10.122 .84 .123 & 8121 & 8121 & 0 & 0 & 0 & 0 \\
\hline 10.122 .84 .133 & 8114 & 8113 & 0 & 0 & 0 & 0 \\
\hline
\end{tabular}

Fig. 18. Comportamiento ante avalancha con ping cada $4 \mathrm{~ms}$.

Para verificar la robustez del sistema se procedió a realizar la misma prueba anterior, pero con tiempos de $1 \mathrm{~ms}$ entre cada pregunta y con una duración de 20 minutos, obteniendo como resultado los datos presentados en la Fig. 19.

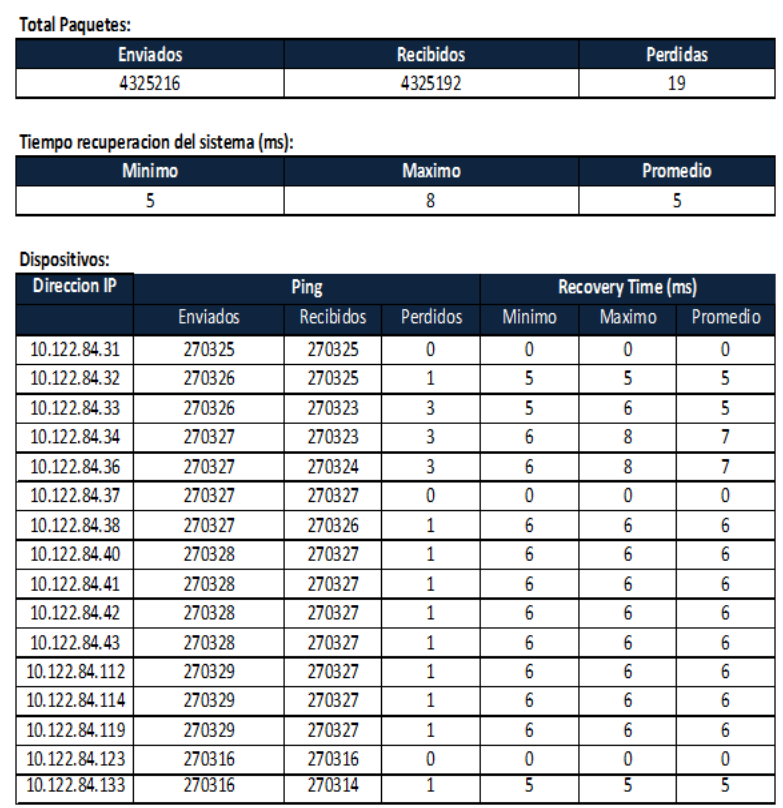

Fig. 19. Comportamiento ante avalancha con ping cada $1 \mathrm{~ms}$.
Durante la prueba se perdieron 19 paquetes y el tiempo de recuperación estuvo entre 5 a $8 \mathrm{~ms}$ en los 13 equipos que presentaron fallas. Durante la prueba existieron tres eventos en los cuales hubo pérdidas de paquetes; en la Fig. 20 se presenta el comportamiento que tuvo cada uno de ellos.

\begin{tabular}{|c|c|c|c|}
\hline Dispositivo & $\begin{array}{c}\text { Ev1 Tiempo de } \\
\text { Recuperacion(ms) }\end{array}$ & $\begin{array}{c}\text { Ev2 Tiempo de } \\
\text { Recuperacion(ms) }\end{array}$ & $\begin{array}{c}\text { Ev3 Tiempo de } \\
\text { Recuperacion(ms) }\end{array}$ \\
\hline 10.122 .84 .31 & 0 & 0 & 0 \\
\hline 10.122 .84 .32 & 5 & 0 & 0 \\
\hline 10.122 .84 .33 & 6 & 5 & 0 \\
\hline 10.122 .84 .34 & 6 & 8 & 0 \\
\hline 10.122 .84 .36 & 6 & 8 & 0 \\
\hline 10.122 .84 .37 & 0 & 0 & 0 \\
\hline 10.122 .84 .38 & 6 & 0 & 0 \\
\hline 10.122 .84 .40 & 6 & 0 & 0 \\
\hline 10.122 .84 .41 & 6 & 0 & 0 \\
\hline 10.122 .84 .42 & 6 & 0 & 0 \\
\hline 10.122 .84 .43 & 6 & 0 & 0 \\
\hline 10.122 .84 .112 & 6 & 0 & 0 \\
\hline 10.122 .84 .114 & 6 & 0 & 0 \\
\hline 10.122 .84 .119 & 6 & 0 & 0 \\
\hline 10.122 .84 .123 & 0 & 0 & 0 \\
\hline 10.122 .84 .133 & 0 & 0 & 5 \\
\hline
\end{tabular}

Fig. 20. Eventos en la red ante avalancha con ping cada $1 \mathrm{~ms}$.

Aunque existieron pérdidas de 19 paquetes durante la prueba, los tiempos de respuesta ante la falla fueron cortos con un máximo de $8 \mathrm{~ms}$. Adicionalmente se generaron eventos en la subestación simultáneamente a la avalancha sin pérdida de ninguna información obteniendo resoluciones menores a $3 \mathrm{~ms}$.

\section{CONCLUSIONES}

La norma IEC 61850 ofrece como ventajas interoperabilidad, flexibilidad, estandarización de ingeniería, conceptos de servicio eficiente, procesos de servicios paralelos, reducción de costos de ingeniería y sistema escalable a nivel de tecnología de comunicación.

Los mensajes GOOSE es una nueva propuesta que permite generar esquemas de protección más robustos, en cuanto a que permite la interacción de todos los dispositivos a nivel de proceso logrando una integridad del sistema $\mathrm{y}$ beneficios en selectividad, confiabilidad y 
eficiencia de la parte eléctrica.

Aunque en principio la aceptación de la norma IEC 61850 tuvo bastantes conjeturas, se pude establecer que el mundo está adoptando esta tecnología teniendo como resultado cerca de 400 sistemas ya implementados a nivel mundial. A nivel local, aunque ya se ha trabajado con la IEC 61850 en las subestaciones más importantes del país, se recomienda a las empresas eléctricas migrar sus sistemas convencionales y entrar al nuevo esquema de sistemas inteligentes a nivel de energía.

Según los conceptos estudiados, para los sistemas eléctricos de potencia implementados con IEC 61850 es importante tener una disponibilidad mayor al 99\%, obteniendo con este valor que 361.35 días del año se garantizara $\mathrm{su}$ buen funcionamiento (confiabilidad del sistema). El valor restante estará dado entre la sumatoria de fallas relacionadas con la duración y frecuencia de las mismas. La comisión de regulación de energía y gas (CREG) estipula estos indicadores dependiendo del tipo de agente del sistema de transmisión nacional (generación, transmisión, distribución, comercialización), por tal motivo es necesario conocer estos indicadores previamente al diseño de acuerdo a los requerimientos del proyecto [19].

El tiempo de transmisión de un sistema en condiciones normales varía aproximadamente de 16 a 28ms, tiempo suficiente para los requerimientos de automatización y protección eléctrica, los cuales oscilan a partir de los $150 \mathrm{~ms}$

\section{Referencias Bibliográficas}

[1] Pacw.org. [En línea], consultado en Junio 2 del 2009, disponible en: http://www. pacw.org/en/issue/ summer_2009_issue/ implementation_strategy/process_bus. html.

[2] A. Behrouz, C. Coombs, S. Chung, Transmisión de datos y redes de comunicaciones, $2^{\mathrm{a}}$ edición, Mc Graw para operación de las funciones de protección.

Desde el punto de vista de las variables de red, se puede concluir que el tiempo de latencia de un mensaje depende básicamente de la topología utilizada, debido a que el Jitter del paquete y del dispositivo de red es constante.

De acuerdo con las pruebas realizadas se concluye que los tiempos de respuesta ante una avalancha en la red cumplen con los requerimientos necesarios para sistemas eléctricos, ya que en promedio están alrededor de los 40ms tiempo permisible para el esquema eléctrico los cuales oscilan desde los 150ms.

Se recomienda la utilización de topología en anillo con RSTP, en lugar de PRP debido a las posibles fallas que éste no puede detectar; en el momento en que en el mercado se tenga PRP/ HSR se recomienda migrar a este sistema, ya que los tiempos de recuperación de RSTP ante una falla hacen que el PRP/HSR sea superior en cuanto a confiabilidad.

Debido a que la implementación de IEC 61850 es basada en la plataforma Ethernet y los sistemas eléctricos de potencia es un factor crítico a nivel mundial, se propone en este artículo como tema de estudio para los lectores interesados en el área, seguridad en cuanto a la información, red y gestión. Haciendo énfasis en la norma NERC CIP (north american electric reliability corporation - critical infrastructure protection), que constituye un conjunto de estándares de cyber-seguridad.
[3] R. Vignoni, R. Pellizzoni, L.Funes, Sistemas de automatización de subestaciones con IEDs IEC 61850, XIII Encuentro Regional Iberoamericano de CIGRÉ, Mayo 2009.

[4] Grupo de trabajo: XM- Compañía de Expertos en Mercados, CIDET (Centro de 
Investigación y Desarrollo Tecnológico del Sector Eléctrico), COCIER (Comité Colombiano de la Comisión de Integración Energética Regional), CNO (Consejo Nacional de Operación), CAC (Comité Asesor de Comercialización) y CINTEL (Centro de Investigación de las Comunicaciones), Marco Estratégico y Propuesta Proyecto Nacional de Redes Inteligentes en Colombia, pp 1-5,6-29, Marzo 2011.

[5] R. Cespedes, Disponibilidad de Subestaciones con Comunicaciones Basadas en el Protocolo IEC-61850, Universidad Nacional de Colombia, Febrero del 2009.

[6] RuggedCom Inc, I 30 Whitmore Road, Woodbridge, Latency on a switched Ethernet network, Application Note 8, April 2008.

[7] T. James, Applying IEEE 802.1w (RSTP) to improve service availability, School of CTI, De Paul University, May 2003.

[8] www.cisco.com. [En línea], consultado en Junio 2 del 2011, disponible en: http://www.cisco.com/en/US/ tech/ tk389/tk621/technologies_white_ paper09186a0080094cfa.shtml.

[9] M. Galea, Rapid spanning tree in Industrial networks, RuggedCom Inc. - Industrial Strength Networks Woodbridge, Ontario, Canada, 2008.

[10] International Standar IEC 61850-5, segunda edición, pp 10-54, 125-130, 2011

[11] International Standar IEC 61850-7-1, segunda edición, pp-20-85, 2011.

[12] International Standar IEC 61850-7-2, segunda edición, pp15-30, 2011.

[13] International Standar IEC 61850-8-1, segunda edición, pp 15-25, 65-80, 2011.

[14] International Standar IEC 61850-3, segunda edición, pp 10-30. 2011.

[15] Pacw.org. [En línea], consultado en Junio 3 de 2011, disponible en: http://www.pacw. org/en/issue/september_2010_issue/ cover_story/ethernet_for_iec_61850/ complete article/1.html.

[16] IEEE 802.1D-2004, Standard for local and metropolitan area networks, Media Access Control (MAC) Bridges, capitulo
17, Junio 2004.

[17] IEC 62439, Committee Draft Vote (CDV): Industrial Communication Networks: High Availability automation networks, capitulo 6, Parallel Redundancy Protocol, Abril 2007.

[18] W. Hans, Parallel Redundancy Protocol (PRP), University of Applied Sciences Institute of Embedded Systems. pp 1-20, Zurich 2009.

[19] creg.gov.co. [En línea], consultado en Julio 4 de 2011, disponible en http:// apolo.creg.gov.co/Publicac.nsf.

[20] J. McGhee; M. Goraj, Smart high voltage substation based on IEC 61850 Process bus and IEEE 1588 time synchronization, 2010 IEEE, May 2010.

[21] C. Maria; P. Ferrari; A. Flammini; S. Rinaldi and M. Quarantelli, On the use of IEEE 1588 in existing IEC 61850-based SAS: Current behavior and future challenges, 2011 IEEE, September 2011.

[22] L. Andersson; Klaus-Peter Brand; C. Brunner and W. Wimmer, Reliability investigations for SA communication architectures based on IEC 61850, 2010 IEEE, January 2010.

[23] A. Daniel, Power quality enhancements for 1EC 61850, 2010 IEEE, 2010.

[24] A. Bonetti; R. Douib, Transfer time measurement for protection relay applications with the IEC 61850 standard, 2010 IEEE, 2010.

[25] R. Cagil; A. Zayegh, and A. Kalam, Object modeling of data and dataSets in the International Standard IEC 61850, 2009 IEEE, july 2009.

[26] D. Ouellette, M. Desjardine; P. Forsyth, Using a real time digital simulator to affect the quality of IEC 61850 GOOSE and sampled value data, 2010 IEEE, 2010.

[27] A. Apostolov, Modeling systems with distributed generators in IEC 61850, 2009 IEEE, 2009.

[28] H. Englert; H. Dawidczak, IEC 61850 substation to control center communication - Status and Practical experiences from projects", 2009 IEEE, 2009.

[29] S. Mohagheghi; Jean-Charles Tournier; J. 
Stoupis; L. Guise; T. Coste; C. Andersen; J. Dall, Applications of IEC 61850 in distribution automation", 2011 IEEE, 2011.

[30] N. Honeth; W. Yiming, N. Etherden; L. Nordström, Application of the IEC 618507-420 data model on a hybrid renewable energy system, 2011 IEEE, 2011.
[31] C. Brunner; IEC 61850 for power system communication, 2008 IEEE, 2008.

[32] R. Mackiewicz, Overview of IEC 61850 and benefits 2006 IEEE, 2006.

[33] IEEE Magazine, IEC 61850 in the oil and gas Industries, IEEE Industry applications magazine, February 2011. 